\title{
Estimates of heterosis for non-production traits for an all dairy breed genetic evaluation in the United Kingdom
}

R Mrode, M Coffey, K Moore, E Wall

Scottish Agricultural College, Penicuik, United Kingdom

Email: raphael.mrode@sac.ac.uk

Introduction Most countries have reported a decline in reproductive traits and other traits associated with overall fitness in recent years as a result of selecting primarily for milk yield. In an attempt to arrest the situation, cross breeding has been embarked upon by many farmers in addition to directly selecting for traits such as fertility (FERT), lifespan (LS) and somatic cell count (SCC). In generally these traits are of low heritability, implying that the gains from heterosis are expected to be larger than those for production traits. While estimates of heterosis for production traits have been reported on United Kingdom (UK) national genetic evaluation data (Mrode et. al., 2008), no such estimates have been reported for non-production traits. This paper summarises the model and estimates of heterosis for FERT, LS and SCC in the UK national genetic evaluation data.

Materials and methods First five parity SCC data and first parity data for fertility traits: Calving interval (CI), non-return rate at day 56 (NR56), test day milk yield at day 110 (MTD), condition score (CS) and days to first service and number of services observed that resulted in a calf, were extracted from the data of milk recording organisations for cows born since 1981. The number of lactations each cow completed (up to the first five parities) and type traits were extracted over the same time period. LS evaluations were then based on a bivariate analysis of lifespan score computed from the number of lactations each cow completed and survival score computed from type information. The fertility index in the UK includes evaluations for CI and NR56 but only the results for CI have been reported in this paper. The breed composition of cows with fertility data consisted of 39\% Holstein (Hol), 0.4\% Friesian (Fri), 1.3\% Ayrshire (Ayr), 2.5\% Jersey (Jer), 0.9\% Guernsey (Gue), less than 1\% for Shorthorn, Brown Swiss, Montebeliarde, Normande and Meuse-Rhine, 38\% crosses among the various pure breeds, with a coefficient of heterosis of at least $50 \%$ and $17 \%$ with a coefficient of heterosis of 25 to $49 \%$. The model for fertility included the fixed effect of herd-year-season (HYS), age (linear and quadratic), days in milk (for MTD only), herd-year-visit (HYV) and stage of parity (STAG) (for CS only). Similarly the model for SCC included the fixed effects of HYS, linear effects of age within parity within breed, month of calving and random effects of animal, herd-by-sire and permanent environment. In the bivariate analysis for LS, the fixed effects were HYS for lifespan score, age (linear and quadratic), milk yield deviated from contemporaries while those for survival score were HYV, STAG, age (linear and quadratic). Random animal effect was fitted for both traits. In all trait groups, linear effects of heterosis and recombination defined for six groups of crosses were fitted in the model. The six crosses were Holstein $\mathrm{x}$ Friesian (Hol $\mathrm{x}$ Fri), Hol x Red and White breeds (Hol x R\&W), with the R\&W consisting of Ayr, Scandinavian Red Brown Swiss, Montebeliarde and Shorthorn, Hol x Others (Jer and Gue), Fri x R\&W, Fri x others and R\&W x Others. Estimates of heterosis are reported for each cross and the predicted transmitting abilities (PTAs) from the all breed analysis correlated with those from within breed analyses.

Results The estimates of heterosis for traits varied across the different crosses and these are presented in Table 1.

Table 1 Estimates of $100 \%$ heterosis for some non-productive traits

\begin{tabular}{lllc}
\hline \hline Breed Cross & Calving Interval (days) & Somatic cell count (\%) & Lifespan (lactations) \\
\hline Hol x Fri & -1.63 & -1.69 & 0.022 \\
Hol x R\&W & -3.13 & -4.50 & 0.170 \\
Hol x Others & -5.73 & -3.70 & 0.114 \\
Fri x R\&W & -2.77 & -3.13 & -0.045 \\
Fri x Others & -2.99 & 0.33 & 0.002 \\
R\&W x Others & -2.32 & 2.00 & 0.142 \\
\hline \hline
\end{tabular}

The estimates of heterosis For CI were largest for the Hol x Others cross with CI being reduced by about 6 days which is 0.11 standard deviation However, the effects of heterosis were largest for SCC and LS in the Hol x R\&W cross with a reduction of about $5 \%$ in SCC in 305d lactation and an increase about 0.2 lactations in a life time. Considering bulls with at least 50 daughters, the correlations of bull PTAs for SCC from the all breed analyses with those from the within breed analysis were $0.98,0.98,0.98$ and 0.96 for Hol, Ayr, Jer and Gue respectively. Corresponding estimates for LS were 0.95 , $0.96,0.94$ and 0.89 respectively. The rate of genetic change per year for SCC from the all breed run was $0.288 \%$ per year with Hol, Fri and various crosses (with at least 25\% coefficient for heterosis), accounting for 50, 14 and $36 \%$ of that rate of change. Similarly, the rate of change for LS was 0.011 lactations per year with Hol, Fri and various crosses accounting for 31,36 and $33 \%$ of the rate of change respectively.

Conclusion Effects of heterosis for the non-production traits when expressed in terms of the standard deviation were slightly less than observed for production. An all breed evaluation for non-production traits in the national data for UK is feasible.

Acknowledgements The authors gratefully acknowledge the input of Marco Winters and DairyCo for funding.

\section{Reference}

Mrode, R., Coffey, M., Sawalha, R. and Wall, E. 2008. Interbull bulletin 30, 48-51 